\title{
Sinc Function Quadrature Rules for the Fourier Integral
}

\author{
By John Lund
}

\begin{abstract}
In this paper a numerical algorithm is proposed for the computation of the Fourier Transform. The quadrature rule developed is based on the Whittaker Cardinal Function expansion of the integrand and a certain Conformal Map. The error of the method is analyzed and numerical results are reported which confirm the accuracy of the quadrature rule.
\end{abstract}

1. Introduction. The numerical computation of the Fourier integral

$$
\hat{f}(\lambda)=\int_{-\infty}^{\infty} f(x) e^{i \lambda x} d x
$$

is beset with difficulties which may arise from the infinite range of integration, the oscillatory integrand or singularities of the function $f$. Since many existing methods and the methods of this paper apply to the real integrals

$$
\hat{g}_{s}(\lambda)=\int_{0}^{\infty} g(x) \sin \lambda x d x
$$

and

$$
\hat{h}_{c}(\lambda)=\int_{0}^{\infty} h(x) \cos \lambda x d x,
$$

it is convenient to rewrite $(1.1)$ as $\hat{f}(\lambda)=i \hat{g}_{s}(\lambda)+\hat{h}_{c}(\lambda)$, where $h(x)=f(x)+f(-x)$ and $g(x)=f(x)-f(-x)$.

Many of the methods employed in the computation of (1.2) (or (1.3)) involve the truncation of the infinite range of integration followed by an application of a classical quadrature rule to the resulting integral with compact support. This method is carried out in [4] with a Gauss-Jacobi quadrature, and various interpolatory rules (trapezoidal, Simpson's, etc.) have been considered in [2] and [5]. These methods all have in common the generation of an alternating sum for the approximation of (1.2) or (1.3), and consequently various acceleration techniques may be brought into play.

Approximating $g(x)$ (or $h(x))$ in (1.2) (or (1.3)), instead of the entire integrand, is probably originally due to Filon [3], where parabolic arcs are used, and various refinements of this procedure have been discussed in [6] and [12]. Expansion of the transformed function in a series of orthogonal functions has been carried out in [9], [13] and [14].

The method of the present paper is based on a Whittaker Cardinal Function expansion of the integrands in (1.2) and (1.3). The method has the advantage that

Received April 7, 1982.

1980 Mathematics Subject Classification. Primary 65D32.

(1)1983 American Mathematical Society 0025-5718/82/0000-1244/\$03.50 
the weights of the quadrature go to zero in an exponential fashion in a neighborhood of the origin, so that the transforms of singular functions can be handled by the method. The weights for increasing values of $x$ are asymptotically equi-spaced, so that the method readily lends itself to an application of an acceleration method.

In Section 2, the rule is developed for functions which decrease rapidly as $x \rightarrow \infty$. Although many existing methods could handle such transforms with equal facility as far as accuracy is concerned, the ease of implementation and its rapid convergence $\left(O\left(e^{-\tau \sqrt{N}}\right), \tau\right.$ a positive constant) as a function of the number of evaluation points $(2 N+1)$ makes the rule highly competitive. Also, the method is conveniently applicable for moderate ranges of the transformed variable $\lambda$. For small $\lambda$ there is no particular difficulty due to the slower oscillation, and for large $\lambda$ the methods of asymptotics can often yield sufficient accuracy (depending on the tolerance required, of course) [8, p. 75]; hence the present method complements existing methods and also deals with singular integrands in a straightforward fashion.

When the function $g$ (or $h$ ) is not rapidly decreasing, the evaluation of its transform is typically more difficult than in the case of the previous paragraph. In the case of slow decrease the cancellation which occurs due to the positive and negative contributions to the integral tends to accentuate errors. It is in the present situation that various "speed-up" techniques may help to sum oscillating series [1, p. 63]. It is often the case that a rule has to be specially tailored to allow for the use of an acceleration technique. In Section 3 it is shown that the application of the Euler transformation in the present case can be carried out in a straightforward fashion with a judicious choice of the mesh size. An algorithm guaranteeing this choice of the mesh size is included in Section 3, and a few test examples (one with a mild singularity) are included which indicate the accuracy of the method.

2. A Conformal Map. Define the region $D_{d}$ in the complex $z=x+i y$ plane

$$
\left\{D_{d}=z|| y \mid<d\right\} \text {, }
$$

where $0<d<\pi / 2$. Assume that $D$ is a simply connected domain in the complex $w=u+i v$ plane and $\phi: D \rightarrow D_{d}$ is a conformal map of $D$ onto $D_{d}$. Further assume that $\phi(a)=-\infty, \phi(b)=+\infty$, where $a$ and $b$ are distinct boundary points of $D$, and set $\Gamma=\left\{x \in D: \phi(x)=w, w \in \mathbf{R}^{\prime}\right\}$. In this situation, the following general quadrature scheme is derived in [10].

TheOREM 2.1. Assume that $f \in \operatorname{Hol}(D)$ and

$$
\int_{\phi^{-1}(x+L)}|f(w) d w| \rightarrow 0 \text { as } x \rightarrow \pm \infty,
$$

where $L=\{i y|| y \mid<d\}$. If $h>0$ and

$$
n_{k}=\phi^{-1}(k h), \quad k=0, \pm 1, \pm 2, \ldots,
$$

then

$$
\begin{aligned}
\int_{\Gamma} f(w) d w-h \sum_{k=-\infty}^{\infty} \frac{f\left(n_{k}\right)}{\phi^{-1}(k h)} \\
\quad=\frac{1}{2} \int_{\partial D} \frac{\exp [i \pi \phi(w) / h] \operatorname{sgn}[\operatorname{Im} \phi(w)]}{\sin (\pi \phi(w) / h)} f(w) d w .
\end{aligned}
$$


The identity (2.3) yields quadrature rules over a large class of contours $T$, as defined above. It furthermore suggests that a given quadrature problem over a contour $\Gamma$ may be dealt with by first transforming the integral over $\Gamma$ into an integral over $\mathbf{R}^{\prime}$, such that (2.1) is satisfied. The form of (2.3) for various maps $\phi$ which will handle the finite, semifinite and infinite real intervals is carried out in [10], and a survey of these formulas (along with many other applications of the Whittaker Cardinal Function) is given in [11].

For oscillatory transforms, and in particular (1.2) and (1.3), specify for $w=u+i v$ the domain $D$ by

$$
D=\{w|| \arg [\sinh (w)] \mid<d\}
$$

Define the map

$$
\phi: D \rightarrow D_{d}
$$

by

$$
\phi(w)=\ln (\sinh (w)),
$$

where "In" denotes the principal value of the natural logarithm. It follows from the Schwarz-Christoffel Theorem that $\phi$ is a conformal map of $D$ onto $D_{d}$ (map the semi-infinite strip into the right half plane and compose with the natural log map).

For what follows it is convenient to set

$$
G(\lambda, x)=g(x) \sin (\lambda x)
$$

and define for $h>0$

$$
n_{k}=\ln \left(e^{k h}+\sqrt{e^{2 k h}+1}\right), \quad k=0, \pm 1, \ldots,
$$

and

$$
W_{k}=\left(1+e^{-2 k h}\right)^{-1 / 2}, \quad k=0, \pm 1, \ldots
$$

TheOrem 2.2. Assume that $g \in \operatorname{Hol}(D)$, that $g$ satisfies (2.1) and

$$
N(g, D)=\lim _{C \rightarrow \partial D} \inf _{C \subseteq D} \int_{C}|g(w) d w|<\infty
$$

If $h>0$, then

$$
\left|d_{s}(g)\right| \equiv\left|\hat{g}_{s}(\lambda)-h \sum_{k=-\infty}^{\infty} W_{k} G\left(\lambda, n_{k}\right)\right| \leqslant \frac{e^{-\pi d / h} \cosh (\lambda d) N(g, d)}{2 \sinh (\pi d / h)} .
$$

Proof. The computation of the weights and nodes (the $W_{k}$ and $n_{k}$, respectively) is a direct application of (2.2) using the map (2.6). Using (2.3) with $f$ replaced by $G(\lambda, w)$ and the identities

$$
\left.\exp \left[\frac{i \pi z}{h}\right] \operatorname{sgn}(\operatorname{Im} z)\right|_{z \in \partial D_{d}}=\exp \left(\frac{-\pi d}{h} \pm \frac{\pi x i}{h}\right)
$$

leads to the inequality

$$
\left|d_{s}(g)\right| \leqslant \frac{e^{-\pi d / h}}{2} \int_{\partial D}\left|\frac{G(, w)}{\sin (\pi \phi(w) / h)}\right| d w
$$


Finally, substituting the two inequalities

$$
\mid \sin \frac{\pi z}{h} \|_{\partial D_{d}} \geqslant \sinh (\pi d / h)
$$

and

$$
\left.|\sin (\lambda w)|\right|_{\partial D_{d}} \leqslant \cosh (\lambda d)
$$

in (2.12) and using (2.10) leads to (2.11). This completes the proof.

To consider the truncation error in (2.11), define the partial sum

$$
S_{-N}^{N}(g)=h \sum_{k=-N}^{N} W_{k} G\left(\lambda, n_{h}\right) .
$$

The conditions (2.1) and (2.10) for arbitrary functions $g$ which are holomorphic in $D$ are not enough to guarantee a rapid convergence of $(2.13)$ to $\hat{g}_{s}(\lambda)$. The convergence is not only dependent on the parameters $\lambda, N$ and $h$ but also on the rate of decrease of the function $g$. For functions of exponential decay the parameter selection and the truncation error are described in the following theorem.

THEOREM 2.3. Assume that $g$ satisfies the hypothesis of Theorem 2.2 and

$$
|g(u)| \leqslant \sigma \begin{cases}u^{\alpha-1}, & u \in(0,1) . \\ e^{-\alpha u}, & u \geqslant 1,\end{cases}
$$

where $\sigma$ and $\alpha$ are positive constants. Then for all $h>0$

$$
\left|S_{-\infty}^{\infty}(g)-S_{-N}^{N}(g)\right| \leqslant \frac{2 \sigma}{\alpha} e^{-\alpha N h}
$$

Proof. The inequality

$$
\left|W_{k}\right| \leqslant \begin{cases}1, & k \geqslant 0, \\ e^{k, h}, & k<0,\end{cases}
$$

is an elementary consequence of the definition (2.9). Using (2.8) and the equality

$$
n_{k}=\int_{0}^{e^{k h}} \frac{d t}{\sqrt{t^{2}+1}}
$$

it follows that

$$
\left|n_{k}\right| \leqslant e^{k h} \quad \forall k
$$

Combining the assumption (2.14) and (2.16) and the inequality

$$
e^{-\alpha n_{h}} \leqslant\left(1+1 / W_{k}\right)^{-\alpha} e^{-\alpha k h}
$$

shows that

$$
\left|W_{k} G\left(\lambda, n_{k}\right)\right| \leqslant \sigma e^{-\alpha k h} \quad \text { if } k \geqslant 0 .
$$

If $k<0$, a comparison of the series expansions of $W_{k}$ and $n_{k}$ (in powers of $e^{k h}$ ) shows that $W_{k} \leqslant n_{k}$. Hence, using (2.14),

$$
\left|W_{k} G\left(\lambda, n_{k}\right)\right| \leqslant \sigma\left(W_{k} n_{k}^{\alpha} / n_{k}\right) \leqslant \sigma n_{k}^{\alpha} \leqslant \sigma e^{\alpha k h} .
$$

It follows that

$$
\left|W_{k} G\left(\lambda, n_{k}\right)\right| \leqslant \sigma e^{-\alpha|k| h} \quad \forall k
$$


Hence

$$
\left|S_{N+1}^{\infty}(g)\right| \leqslant h \sum_{h=N+1}^{\infty} e^{-\alpha k h}=\frac{h e^{-(N+1) h \alpha}}{1-e^{-\alpha h}} \leqslant \frac{\sigma}{\alpha} e^{-N \alpha h}
$$

since $\alpha h \leqslant e^{\alpha h}-1$. The sum $S_{-N}^{-x}$ is similarly bounded, and (2.15) follows from the triangle inequality. This completes the proof.

Combining (2.11) and (2.15) it follows that

$$
\left|\hat{g}_{s}(\lambda)-S_{-N}^{N}(g)\right| \leqslant E_{T}+E_{C},
$$

where

$$
E_{T}=\frac{2 \sigma}{\alpha}\left(e^{-\alpha N h}\right)
$$

and

$$
E_{C}=e^{-\pi d / h}\left(\frac{\cosh (\lambda d)}{2 \sinh (\pi d / h)} \cdot N(g, D)\right)
$$

The selection

$$
h=(2 \pi d / \alpha N)^{1 / 2}
$$

in the truncation error bound $E_{T}$ and the contour error integration bound $E_{C}$ is, for each fixed $\lambda, O\left(\exp \left(-(2 \pi d \alpha N)^{1 / 2}\right)\right)$. However, for moderate and large values of $\lambda$ a more judicious choice of the mesh size $h$ can be made. In particular, given $\lambda$ and an error tolerance $10^{-\beta}\left(\beta \in Z^{+}\right)$, select

$$
h=2 \pi d /(\lambda d+\beta \ln 10)
$$

and

$$
N=[|(\beta \ln 10) / \alpha h+1|],
$$

where $[|x|]$ is the integer part of $x$. These are the selections listed in the table below for Example (2.1).

EXAMPLE (2.1)

$$
\hat{g}_{s}(\lambda)=\int_{0}^{x} e^{-x} \sin \lambda x d x
$$

Accuracy requested $\simeq C \cdot 10^{-4}$

$\begin{array}{rcccc}\lambda & h & N=M & \text { A. E. } & \text { R. E. } \\ 1 & .92 & 11 & 8 . \times 10^{-5} & 1 . \times 10^{-4} \\ 5 & .58 & 16 & 6 . \times 10^{-5} & 2 . \times 10^{-4} \\ 10 & .40 & 25 & 4 . \times 10^{-5} & 4 . \times 10^{-4} \\ 25 & .20 & 46 & 7 . \times 10^{-6} & 1 . \times 10^{-4} \\ 50 & .11 & 82 & 8 . \times 10^{-6} & 4 . \times 10^{-4}\end{array}$

\begin{tabular}{rrrll}
\multicolumn{5}{c}{ Accuracy requested $\simeq C \cdot 10^{-8}$} \\
1 & .49 & 38 & $5 . \times 10^{-10}$ & $1 . \times 10^{-9}$ \\
5 & .38 & 50 & $7 . \times 10^{-9}$ & $3 . \times 10^{-8}$ \\
10 & .29 & 65 & $1 . \times 10^{-11}$ & $1 . \times 10^{-10}$ \\
25 & .17 & 109 & $1 . \times 10^{-9}$ & $3 . \times 10^{-8}$ \\
50 & .10 & 182 & $5 . \times 10^{-10}$ & $6 . \times 10^{-8}$
\end{tabular}


For this example, the selection of the parameters $h$ and $N$ are given by (2.23), (2.24), respectively. The last column in the table is the relative error. The errors are obtained from the known transform $\hat{g}_{s}(\lambda)=\lambda /\left(\lambda^{2}+1\right)$.

Before turning to the examples however, note that, in cases where $g$ is singular at $x=0\left(g^{\prime}(x)\right.$ does not exist at $\left.x=0\right)$, the use of a symmetric approximating sum $S_{-N}^{N}(g)$ to compute $\hat{g}_{s}(\lambda)$ is not the most efficient method of evaluating $\hat{g}_{s}(\lambda)$. In particular, if $|g(u)| \leqslant \sigma u^{\gamma-1}(\gamma>0)$ in (2.14) and $S_{-N}^{N}(g)$ is replaced by $S_{-M}^{N}(g)$, then the truncation error $E_{T}$ in (2.21) takes the form

$$
E_{T}=\sigma\left(e^{-\gamma M h} / \gamma+e^{-\gamma N h} / \alpha\right) \text {. }
$$

Here the selections (2.23) and (2.24) are maintained, and the lower index of summation is selected according to

$$
M=[|\alpha N / \gamma|] .
$$

If $g$ is singular at the origin, the selection (2.26) has the effect of sampling the function $g$ more frequently in a neighborhood of the origin. This can be seen in Example (2.2) below where $M=2 N$.

EXAMPLE (2.2)

$$
\hat{g}_{s}(\lambda)=\int_{0}^{x} \frac{e^{-x} \sin \lambda x}{\sqrt{x}} d x
$$

$$
\text { Accuracy requested } \simeq C \cdot 10^{-4}
$$

$\begin{array}{rlccc}\lambda & h & N & \text { A. E. } & \text { R. E. } \\ 1 & .92 & 10 & 7 . \times 10^{-5} & 1 . \times 10^{-4} \\ 5 & .58 & 15 & 2 . \times 10^{-5} & 5 . \times 10^{-5} \\ 10 & .40 & 25 & 4 . \times 10^{-5} & 1 . \times 10^{-4} \\ 25 & .20 & 46 & 2 . \times 10^{-6} & 1 . \times 10^{-5} \\ 50 & .11 & 82 & 3 . \times 10^{-6} & 2 . \times 10^{-5}\end{array}$

\begin{tabular}{rrrll}
\multicolumn{5}{c}{ Accuracy requested $\simeq C \cdot 10^{-8}$} \\
1 & .49 & 37 & $3 . \times 10^{-8}$ & $6 . \times 10^{-8}$ \\
5 & .38 & 49 & $4 . \times 10^{-9}$ & $9 . \times 10^{-9}$ \\
10 & .29 & 65 & $2 . \times 10^{-9}$ & $5 . \times 10^{-9}$ \\
25 & .17 & 109 & $1 . \times 10^{-9}$ & $5 . \times 10^{-9}$ \\
50 & .10 & 182 & $3 . \times 10^{-10}$ & $2 . \times 10^{-9}$
\end{tabular}

For this example, the selection of $h, N$ and $M$ are given by (2.23), (2.24) and (2.26), respectively. The absolute and relative errors are obtained using the known transform $\hat{g}_{s}(\lambda)=\sqrt{\pi / 2}(Q-1)^{1 / 2} / Q$ where $Q=\left(\lambda^{2}+1\right)^{1 / 2}$.

A word of caution should be included with regard to the computation of the nodes $n_{k}$ in (2.8). Since the accuracy of the error in (2.20) depends upon an accurate computation of the integrand functional values, it is important to compute these nodes accurately in a neighborhood of the origin (especially in the case of singular functions $g$, as in Example (2.2)). Due to round-off, direct use of (2.8) is ill-advised, 
and the following procedure is what was used in the examples of this paper. Rewrite (2.8) in the form

$$
n_{k}=e^{k h}-\frac{1}{6} e^{3 k h}+\frac{3}{40} e^{5 k h}-\frac{15}{336} e^{7 k h}+O\left(e^{9 k h}\right),
$$

and note that for $e^{k h} \in(0,1)$ one obtains values for the $n_{k}$ with ten places of accuracy if the first four terms on the right-hand side of (2.9) are used.

The result of Theorem (2.3) corresponding to the cosine transform (1.3) is summarized in the following theorem.

Theorem 2.4. Assume $h$ in (1.3) satisfies the hypothesis of Theorem (2.2) and the inequality (2.14). Define

$$
H(\lambda, x)=h(x) \cos \lambda x
$$

Then

$$
\left|h_{c}(\lambda)-h \sum_{k=-N}^{N} W_{k} H\left(\lambda, n_{k}\right)\right| \leqslant \frac{2 \sigma}{\alpha} e^{-\alpha N h}+E_{C}
$$

where $E_{C}$ is defined in $(2.22)$ with $N(h, D)$ replacing $N(g, D)$.

3. Functions of Slow Decrease. The assumption (2.14) of the last section severely limits the number of transforms that may be computed using the approximation in (2.13). Although the infinite sum in (2.11) is very accurate for functions $g$ which do not satisfy (2.14), the number of function evaluations required to obtain this accuracy by directly summing (2.13) can be prohibitively large. In this section, it is shown that an accurate approximation of the transforms (1.2) and (1.3) may be obtained from (2.13) if the Euler Transformation is judiciously applied to the approximating sum (2.13). As in Section 2, the details of the quadrature rule will be carried out for the sine transform, and the necessary changes for the cosine transform will be summarized at the end of this section.

Rewrite the approximating sum (2.13) in the form

$$
S_{-M}^{\infty}(g)=h \sum_{k=-M}^{\infty} W_{k} G\left(\lambda, n_{k}\right)=S_{-M}^{K-1}(g)+S_{K}^{\infty}(g),
$$

where $0<K<N$ will be specified in what follows. $G(\lambda, x)$ and $W_{k}$ are defined by (2.7) and (2.9), respectively. Using the inequalities (2.14), (2.17) and (2.18), it follows that

$$
\left|S_{-\infty}^{-M-1}(g)\right| \leqslant \sigma e^{-\alpha M h} / \alpha .
$$

Hence the truncation of the sum $S_{-\infty}^{K-1}$ produces no difficulty, and the first sum on the right-hand side of (3.1) may be summed directly.

Instead of summing $S_{K}^{\infty}(g)$ in (3.1) directly, define

$$
I_{\delta}=\{x \mid \delta \pi / \lambda \leqslant x<(\delta+1) \pi / \lambda\}
$$

and

$$
\alpha_{\delta}=\left|h \sum_{n_{k} \in I_{\delta}} W_{k} G\left(\lambda, n_{k}\right)\right| \text {, }
$$


so that the sum $S_{K}^{\infty}(g)$ can be rewritten as

$$
S_{K}^{\infty}(g)=\sum_{\delta \geqslant K}^{\infty}(-1)^{\delta} \alpha_{\delta} .
$$

Now (3.5) is an alternating series, and it is known that the Euler method of summation applied to such series often accelerates their convergence. Explicit conditions on the terms $\alpha_{\delta}$ concerning when such an acceleration is effected may be found in [1, p. 63]. For the remainder of this section all that need be assumed is that there exists a function

$$
k(x)=(x+a)^{-b}, \quad a, b>0,
$$

such that $k\left(x_{\delta}\right)=\alpha_{\delta}$ and the $x_{\delta}$ are equispaced. If the sum in (3.5) is denoted by $S$ and the truncation errors in approximating $S$ by the partial sums

$$
S_{K}^{N}(g)=\sum_{k=K}^{N}(-1)^{\delta} \alpha_{\delta} \text { and }(-1)^{K} \sum_{k=0}^{N-K} \frac{(-1)^{k} \Delta^{k} \alpha_{K}}{2^{k+1}}
$$

are denoted by $E_{N}$ and $E_{N}^{T}$, respectively, then $\lim _{N \rightarrow x}\left(E_{N}^{T} / E_{N}\right)=0$. Moreover, the error in approximating the sum $S$ by the transformed sum satisfies

$$
\left|S-(-1)^{K} \sum_{k=0}^{N-K} \frac{(-1)^{k} \Delta^{k} \alpha_{K}}{2^{k+1}}\right| \leqslant \frac{\left|\Delta^{N-K} \alpha_{K}\right|}{2^{N-K+1}} .
$$

Assuming that the $\alpha_{\delta}$ in (3.4) satisfy the conditions outlined in the above, the following theorem has been established.

THEOREM 3.1. Assume $g$ in (1.2) satisfies the hypothesis of Theorem (2.2) and

$$
|g(u)| \leqslant \sigma u^{\alpha \cdot 1}, \quad u \in(0,1) .
$$

If the selection

$$
h=\frac{\pi}{\gamma \lambda}, \quad \gamma \in Z^{+}
$$

is used in (3.1), then

$$
\begin{array}{r}
\left|\hat{g}_{s}(\lambda)-S_{-M}^{K-1}(g)-(-1)^{K} \sum_{k=0}^{N} \frac{(-1)^{k} \Delta^{k} \alpha_{K}}{2^{k+1}}\right| \\
\leqslant \frac{\sigma e^{-\alpha M h}}{\alpha}+C e^{-(2 \gamma-1) \lambda d}+\frac{\left|\Delta^{N-K} \alpha_{K}\right|}{2^{N-K+1}},
\end{array}
$$

where $C$ is a constant depending on $g$ and $h$.

Now if it is required to compute $\hat{g}_{s}(\lambda)$ to within a tolerance of $10^{-\beta}$, the following selection of the parameters $M, \gamma, K, N$ and the inequality (3.10) guarantee that the accuracy is achieved. For $\gamma$ in (3.9), put

$$
\gamma=\left[\left|\frac{\beta \ln 10}{2 \lambda d}+1\right|\right]
$$

and set

$$
M=\left[\left|\frac{\beta \ln 10}{\alpha h}\right|\right]
$$


These selections guarantee that the first two terms on the right-hand side of $(3.10)$ are bounded by a constant times $10^{-\beta}$. In practice, one has little control over the function $g$ being transformed, so that the selection of the parameters $K$ and $N$ are predicated on the other factors which are typically associated with the acceleration of the convergence of a series when the Euler Transformation is used (equispaced sampling of $k$ in (3.6) and "small" differences of the $\alpha_{\delta}$ in (3.4)). The selection

$$
K=\left[\mid \frac{\beta \ln 10}{2 h} \|\right]
$$

in (3.7) guarantees that the nodes $n_{k}$ are asymptotically equispaced to within the accuracy required of the computation, i.e.

$$
n_{k}=k h+\ln 2+O\left(e^{-2 k h}\right) \text {. }
$$

With the selection of $h$ in (3.9) there are $\gamma$ nodes between consecutive zeros of $\sin (\lambda x)$, so that the selection

$$
\begin{aligned}
& N=p \gamma+K, \\
& p=[|\beta(\ln 10+1)|]
\end{aligned}
$$

in (3.7) insures that a sufficient number of terms have been taken in the transformed sum to satisfy the inequality in (3.7). With these selections, it is guaranteed that the right-hand side of (3.10) is bounded above by a constant times $10^{-\beta}$.

Although the selection of the parameters discussed in the preceding paragraph show that $\hat{g}_{s}(\lambda)$ may be computed to within a given tolerance, the author has found that the selections are quite pessimistic in practice. Before discussing the numerical implementation of (3.10), its analogue for the cosine transform (1.3) is summed up in the following theorem.

THEOREM 3.2. Assume that $h$ in (1.3) satisfies the hypothesis of Theorem (3.1) and the inequality (3.8). If the selection $h$ in (3.9) is made, then

$$
\begin{gathered}
\left|\hat{h}_{c}(\lambda)-S_{-M}^{K-1}(h)-(-1)^{K} \sum_{k=0}^{N-K} \frac{(-1)^{k} \Delta^{k} \alpha_{K}}{2^{k+1}}\right| \\
\leqslant \frac{\sigma}{\alpha} e^{-\alpha M h}+C e^{-(2 \gamma-1) \lambda d}+\frac{\left|\Delta^{N-K} \alpha_{K}\right|}{2^{N-K+1}} .
\end{gathered}
$$

Here the partial sum $S_{-M}^{K-1}(k)$ and the $\alpha_{k}$ are the analogues of (3.1) and (3.4) for the cosine kernel. Also, the discussion which follows Theorem (3.1) concerning the selection of the parameters for (3.10) applies verbatim to (3.16). The implementation of the approximation in (3.10), which follows, also applies to the approximation in (3.16).

In practice, the author has found that the selections (3.9), (3.11) and (3.12) cannot be improved upon for a large number of examples computed. These are the selections made in each of the two examples listed below. However, the selections of the "cutoff" $K$ and the number of terms, $p$, to which the Euler Transformation is applied are far too pessimistic. As a case in point here, consider $\lambda=10$ in Example (3.1) below where an accuracy of $10^{-8}$ was given as a tolerance. The selection (3.13) and (3.15) give $K=29$ and $N=47$, respectively. A reference to the table in Example (3.1) shows that the requested accuracy was obtained with $K=8$ and 
$N=18$. This is not an isolated case and is to be somewhat expected in view of the fact that (3.13) and (3.15) do not take into account the relative size of $\left|\Delta^{N-K} \alpha_{K}\right|$, which for sequences generated by functions of the form in (3.6) (and many others) can be quite small. What is done in practice is the following: select $p \in(0,1]$ ( 1 is used in the examples below) and the cutoff $K$ by

$$
\left|g\left(x_{K}\right)\right| \leqslant p / \gamma,
$$

where $\gamma$ is defined in (3.11). This selection, in general, guarantees that the differencing in the Euler Transformation does not begin until the differences $\Delta^{k} \alpha_{K}$ are small.

EXAMPLE (3.1)

$$
\hat{g}_{s}(\lambda)=\int_{0}^{x} \frac{\sin \lambda x}{x+1} d x
$$

Accuracy requested $\simeq C \cdot 10^{-4}$

$\begin{array}{rcrrccc}\lambda & h & M & K & \left(\begin{array}{c}\text { \# of terms } \\ \text { Eulered }\end{array}\right) & \text { A. E. } & \text { R. E. } \\ 1 & \pi / 3 & 8 & 5 & 6 & 4 . \times 10^{-5} & 6 . \times 10^{-5} \\ 5 & \pi / 5 & 14 & 6 & 9 & 1 . \times 10^{-4} & 8 . \times 10^{-4} \\ 10 & \pi / 10 & 29 & 8 & 6 & 5 . \times 10^{-7} & 1 . \times 10^{-6} \\ 25 & \pi / 25 & 73 & 19 & 10 & 1 . \times 10^{-7} & 3 . \times 10^{-6} \\ 50 & \pi / 50 & 146 & 36 & 8 & 2 . \times 10^{-7} & 1 . \times 10^{-5}\end{array}$

Accuracy requested $\simeq C \cdot 10^{-8}$

$\begin{array}{rcrrrll}1 & \pi / 6 & 35 & 5 & 9 & 1 . \times 10^{-7} & 2 . \times 10^{-7} \\ 5 & \pi / 10 & 58 & 12 & 9 & 4 . \times 10^{-9} & 2 . \times 10^{-8} \\ 10 & \pi / 10 & 58 & 8 & 10 & 2 . \times 10^{-8} & 2 . \times 10^{-7} \\ 25 & \pi / 25 & 146 & 19 & 10 & 1 . \times 10^{-9} & 2 . \times 10^{-8} \\ 50 & \pi / 50 & 292 & 36 & 8 & 1 . \times 10^{-10} & 5 . \times 10^{-9}\end{array}$

For this example, the selections for $h, M$, and $K$ are given by (3.9), (3.12) and (3.17), respectively. The absolute and relative errors are obtained from known values of $\hat{g}_{s}(\lambda)$ given in terms of Sine and Cosine integrals.

$$
\begin{gathered}
\text { Example }(3.2) \\
\hat{g}_{s}(\lambda)=\int_{0}^{\infty} \sqrt{x} \frac{\sin \lambda x}{x+1} d x
\end{gathered}
$$

Accuracy requested $\simeq C \cdot 10^{-4}$

$\begin{array}{ccrrccc}\lambda & h & M & K & \left.\begin{array}{c}\# \text { of terms } \\ \text { Eulered }\end{array}\right) & \text { A. E. } & \text { R. E. } \\ \pi / 2 & \pi / 2 \lambda & 6 & 7 & 3 & 3 . \times 10^{-4} & 1 . \times 10^{-3} \\ 2 \pi & \pi / \lambda & 12 & 10 & 4 & 2 . \times 10^{-5} & 5 . \times 10^{-4} \\ \frac{25 \pi}{8} & \pi / \lambda & 19 & 10 & 9 & 6 . \times 10^{-7} & 2 . \times 10^{-5} \\ 8 \pi & \pi / \lambda & 49 & 27 & 9 & 2 . \times 10^{-6} & 4 . \times 10^{-4} \\ \frac{25 \pi}{2} & \pi / \lambda & 76 & 42 & 9 & 3 . \times 10^{-6} & 1 . \times 10^{-3}\end{array}$




\begin{tabular}{ccccccc}
\multicolumn{7}{c}{ Accuracy requested $\simeq C \cdot 10^{-8}$} \\
$\pi / 2$ & $\pi / 4 \lambda$ & 24 & 20 & 9 & $1 . \times 10^{-8}$ & $4 . \times 10^{-8}$ \\
$2 \pi$ & $\pi / \lambda$ & 24 & 24 & 9 & $2 . \times 10^{-8}$ & $2 . \times 10^{-7}$ \\
$\frac{25 \pi}{8}$ & $\pi / \lambda$ & 38 & 15 & 9 & $7 . \times 10^{-8}$ & $3 . \times 10^{-6}$ \\
$8 \pi$ & $\pi / \lambda$ & 98 & 27 & 9 & $8 . \times 10^{-10}$ & $1 . \times 10^{-7}$ \\
$\frac{25 \pi}{2}$ & $\pi / \lambda$ & 152 & 42 & 9 & $2 . \times 10^{-10}$ & $7 . \times 10^{-8}$
\end{tabular}

For this example, the selections for $h, M$, and $K$ are given by (3.9), (3.12) and (3.17), respectively. The absolute and relative errors are obtained from known values of $\hat{g}_{s}(\lambda)$ given in terms of the Fresnel Integrals.

Acknowledgement. The author would like to thank his advisor Professor Frank Stenger (University of Utah) for his guidance during the writing of the author's Ph.D. thesis. This paper was a part of that thesis. The author is also grateful to $\mathrm{Mr}$. Paul Jacobs for his programming assistance.

Department of Mathematical Sciences

Montana State University

Bozcman. Montana 59717

1. T. S. Bromwich, An Introduction to the Theory of Infinite Series, Macmillan. New York, 1966.

2. (i. Debalbine \& J. N. Franklin, "The calculation of Fourier Integrals," Math. Comp., v. 20, 1966, pp. 57()$-589$.

3. L. N. Filon, "On a quadrature formula for trigonometric integrals," Proc. Rol. Soc. Edinburgh, v. 49. 1929. pp. $38-47$.

4. M. MURwitz. JR. \& P. F. ZWEIFEL, "Numerical quadrature of Fourier transform integrals," MT.4C.v. 10. 1956, pp. 140-149.

5. V. I. Krylov \& N. S. Skoblya, Handbook of Numerical Ineersion of Laplace Transforms, Israel Program for Sci. Transl.. Jerusalem. 1969.

6. Y. L. Luke. "On the computation of oscillatory integrals," Proc. Cambridge Philos. Soc., v. 50. 1954. pp. 269-277.

7. M. K. Miller \& W. T. GuY, JR.. "Numerical inversion of the Laplace transform by use of Jacobi polynomials," SIAM J. Numer. Anal., v. 3. 1966, pp. 624-635.

8. F. W. J. Olver. Introduction to Așimptotics and Special Functions, Academic Press, New York, 1974.

9. M. E. SALzER, "Orthogonal polynomials arising in the numerical evaluations of inverse Laplace transforms," MTAC. v. 9. 1955. pp. 164-177.

1). F. Stenger. "Integration rules via the trapezoid formula." J. Inst. Math. Appl., v. 12, 1973, pp. 1() $3-114$

11. F. Stenger, "Numerical methods based on Whittaker cardinal, or sinc functions," SIAM Rer., v. 23. 1981. pp. 165-224.

12. A. J. VAN DE VOOREN \& M. J. VAN LINDE, "Numerical calculation of integrals with strongly oscillating integrands," Math. Comp., v. 20. 1966. pp. 232-245.

13. (j. Walter \& D. Schultz, "Some eigenfunction methods for computing a numerical Fourier transform." J. Inst. Math. Appl., v. 18, 1976, pp. 279-293.

14. M. WeBER. "Numerical computation of the Fourier transform using Laguerre functions and the fast Fourier transform.” Numer. Math., v. 36, 1981, pp. 197-209. 